\title{
Análisis de la difusión de la internet móvil en España
}

\author{
María Rosalía Vicente* \\ Departamento de Economía Aplicada \\ Universidad de Oviedo
}

\section{Resumen}

Los últimos años han sido testigos de cómo se han ido popularizando toda una serie de dispositivos digitales (teléfonos, agendas electrónicas, ordenadores portátiles, mini-ordenadores, e-books) que permiten acceder a Internet de forma móvil, desde cualquier lugar y en cualquier momento. Usando los datos de la última encuesta del INE sobre el uso TIC en España, este trabajo tiene por objeto analizar la utilización de tales dispositivos móviles entre la población, identificando cuáles son los factores de tipo socio-económico y demográfico que determinan que un individuo decida emplear este tipo de tecnología para acceder a Internet.

Palabras clave: internet, difusión tecnológica, acceso móvil, tecnologías de la información y la comunicación (TIC), España.

Clasificación JEL: O33; O52; L86; L96.

\section{Abstract}

The last years have witnessed the growth in the use of several digital gadgets (telephones, electronic agendas, laptops, mini-computers, e-books) which allow to access the Internet "on the go», from anywhere and at any time. Using data from the last survey on ICT usage collected by the Spanish NIS, the aim of this paper is to analyse the take-up of the mobile Internet in Spain, identifying the socio-economic and demographich features which significantly influence an individual's decision on using this kind of technology to access the Internet.

Keywords: internet, technological diffusion, mobile access, information and communication technologies (ICT), Spain.

JEL classification: O33; O52; L86; L96

\section{Introducción}

2010 ha sido el año del lanzamiento de dos nuevos dispositivos digitales sobre los que existía gran expectacion: el ipad y el iphone 4, ambos a cargo de la empresa Apple. Si algo ha caracterizado el sector de las tecnologías de la información y la comunicación (TIC) a lo largo de los últimos años es su rapidísima evolución tecnólogica. De hecho, la conocida como ley de Moore establece que «cada 18 meses se duplica la potencia de los microprocesadores».

* La autora quiere agradecer los comentarios recibidos de un revisor anónimo que han contribuido a mejorar este trabajo. 
Este veloz ritmo de progreso tecnólogico ha hecho que el foco de atención en el análisis de la difusión de estas tecnologías también se haya ido desplazando. Si al principio, la cuestión clave estaba en el acceso a Internet, en si ese acceso se realizaba desde el hogar y, con ello, en estudiar la brecha que había entre los que sí tenían acceso y aquéllos que no; a medida que los niveles de penetración han ido aumentando en todos los países, la atención se ha desplazado hacia asuntos tales como la rapidez de las conexiones, la bancha ancha, los servicios y aplicaciones que los individuos están utilizando online, sus competencias digitales, y el uso de la Internet móvil (DiMaggio et al., 2004; Comisión Europea, 2008; OCDE, 2009).

De la importancia de tales elementos da cuenta la Agenda Digital que ha definido la Unión Europea como uno de los instrumentos básicos de la estrategia de crecimiento para la próxima década, «Europa 2020». En este sentido, se han fijado como objetivos claves el logro de la cobertura universal de la banda ancha básica para 2013, garantizando que para finales de la década un 50 por 100 de los hogares europeos cuente ya con unas conexiones con velocidades por encima de 100 megabits por segundo (Mbps), y el 50 por 100 restante no baje de un nivel mínimo de 30Mbps (Comisión Europea, 2010b). Se han destinado además 18 millones de euros para la investigación en una nueva generación de redes que permitan una Internet móvil de muy alta velocidad, ya que como apunta la Comisión Europea la nueva revolución de Internet es ante todo una revolución de convergencia y movilidad, en el sentido de que «los servicios convergen y pasan del mundo físico al mundo digital accesible universalmente desde cualquier dispositivo, sea un teléfono inteligente (...), un ordenador personal, una radio digital...» (Comisión Europea, 2010b).

De hecho, el mayor ritmo de crecimiento se viene observando en la difusión de la banda ancha móvil, que alcanzó una tasa de penetración en el conjunto de la Unión Europea del 6,1 por 100 en julio de 2010, lo que representa un aumento de casi el 50 por 100 respecto al mismo mes del año anterior. Sin embargo, como ya ha ocurrido con otras tecnologías de la misma rama, su difusión dista de seguir un patrón equilibrado entre los estados miembros, con cifras de penetración que van desde el 1,5 por 100 de Chipre hasta el 16,7 por 100 de Austria y el 21,5 por 100 de Finlandia, pasando por el 7,5 por 100 de España ${ }^{1}$ (Comisión Europea, 2010a).

En este contexto, el objetivo de este trabajo consiste en analizar cómo se está produciendo la difusión de la Internet móvil en España, o dicho de otra forma, del uso de dispositivos móviles para acceder a Internet. Tal como señalan Karshenas y Stoneman (1995), todo proceso de difusión comprende dos grandes decisiones: la primera, la decisión de adopción de la tecnología, es decir, de comenzar a utilizarla; y, la segunda, para aquéllos que ya son usuarios, la intensidad de uso. Este trabajo se centra en la primera. Así, utilizando los datos de la última encuesta del INE sobre el uso TIC en los hogares, se tratará de identificar cúales son los factores de tipo socioeconómico y demográfico que determinan que un individuo decida emplear este tipo

${ }^{1}$ Estas cifras se refieren a las líneas de acceso de banda ancha móvil mediante llaves USB o tarjetas de datos por cada 100 ciudadanos. 
de tecnología. Si bien existen algunos estudios previos para los casos de los Estados Unidos (Smith, 2010) y Japón (Ishii, 2004; Akiyoshi y Ono, 2008), este es un campo aún por abordar tanto en la Unión Europea como en España.

Con tal objetivo, el artículo se estructura en las siguientes partes: se realiza primero una revisión de la literatura sobre los factores tanto a nivel micro como a nivel macro que explican la difusión TIC; a continuación se describen la base de datos empleada para el análisis del uso móvil de Internet en España así como la metodología a utilizar, para luego analizar los resultados obtenidos en las estimaciones y finalmente presentar algunas conclusiones.

\section{Revisión de la literatura}

Las tecnologías de la información y la comunicación han sido las protagonistas absolutas del enorme proceso de transformación económica y social vivida durante los últimos años. Como consecuencia se ha desarrollado una intensa actividad investigadora centrada en el análisis del proceso de difusión de estas tecnologías y, más concretamente, en determinar cuáles son los factores que explican su adopción y uso. Dentro de esta literatura pueden distinguirse dos grandes líneas de investigación: la primera, desde una perspectiva macroeconómica, trata de explicar el proceso de difusión international de las TIC (Hargittai, 1999; Caselli y Coleman, 2001; Kiiski y Pohjola, 2002; Beilock y Dimitrova, 2003; Pohjola, 2003; Dasgupta et al., 2005; Guillén y Suárez, 2005; Chinn y Fairlie, 2007, 2010; Andrés et al., 2010), y la segunda, desde un enfoque microeconómico, presta atención a su utilización por parte de la población (US Department of Commerce, 2000; 2010; Fairlie, 2004; Demoussis y Giannakopoulos, 2006; Horrigan et al., 2006; Vicente y López, 2006; Schleife, 2010). Los resultados de ambas líneas apuntan en la misma dirección al señalar como variables explicativas claves las características socio-económicas de la población (renta, nivel educativo, edad, hábitat) junto con las externalidades de red. A nivel macro, el contexto regulatorio e institucional también juega un papel fundamental.

La renta es así el factor más importante para explicar la adopción de las TIC (Hargittai, 1999; Norris, 2001; Kiiski y Pohjola, 2002; Pohjola, 2003; ITU, 2006; Chinn y Fairlie, 2007, 2010; Hilbert, 2010). En concreto, las estimaciones de Chinn y Fairlie (2010), sobre un panel de 161 naciones, indican que las diferencias de renta explican prácticamente la mitad de la brecha que existe a nivel mundial en las tasas de penetración de Internet.

Las restricciones financieras son igualmente la causa de que los individuos en situación de desempleo o los territorios con mayores tasas de paro tengan menores niveles de adopción (Billón et al., 2008), a pesar de la creciente importancia de Internet para la búsqueda de empleo (Schleife, 2010).

De igual forma el nivel educativo de la población es un factor fundamental para entender el proceso de difusión de este tipo de tecnologías, que a diferencia de otras, 
requieren un alto grado de interacción por parte del usuario (Rogers, 2003), quien además debe contar con las competencias necesarias para poder manejarse en un contexto de gran abundancia de información y evitar que tal riqueza «se transforme en una pobreza de atención» tal como señalaba el Nobel de Economía Herbert Simon (Shapiro y Varian, 1999). En este sentido, existe una amplia evidencia empírica tanto a nivel micro como a nivel macro del impacto positivo del capital humano sobre la adopción TIC (Hargittai, 1999; Norris, 2001; Vicente y López, 2006; Chinn y Fairlie, 2007, 2010; Billón et al., 2008, 2009).

No obstante, algunos estudios recientes apuntan que tales obstaculos económicos y educativos están perdiendo vigor con la popularización de ciertos dipositivos móviles y su utilización para navegar por Internet. Así, por ejemplo, el trabajo de Akiyoshi y Ono (2008), pone de manifiesto que la renta no es un factor significativo a la hora de explicar el acceso a Internet de la población japonesa desde el teléfono móvil. En la misma línea, las cifras del último informe Pew Internet (Smith, 2010) indican que este tipo de conexión es más frecuente entre colectivos tradicionalmente desfavorecidos como los latinos y afro-americanos; sin embargo, las barreras seguirían manteniéndose para el caso de los ordenadores portátiles.

La edad es asimismo un elemento clave para entender el mayor o menor grado de difusión de estas tecnologías entre la población. En general, el uso de las TIC es especialmente intensivo entre las generaciones más jóvenes nacidas ya dentro de la era digital (Fairlie, 2004; Vicente y López, 2006, 2010). Son los llamados «nativos digitales», acostumbrados desde niños a interactuar con estas tecnologías y que tienen en Internet su primera fuente de informacion, frente a los «inmigrantes digitales», éstos son, los adultos que han tenido que aprender a desenvolverse en el mundo digital (Prensky, 2001; Helsper y Eynon, 2010).

Otro de los factores que se ha considerado para explicar la difusión TIC es el tipo de hábitat. Las zonas urbanas disponen normalmente de más recursos y con ellos, de mejores infraestructuras y de redes más rápidas que las áreas rurales, lo que hace que los individuos de las primeras tengan más incentivos a conectarse (US Department of Commerce, 2000; Grubesic, 2006; Billón et al., 2008). No obstante, conviene señalar los esfuerzos institucionales que se han realizado durante los últimos años destinados a cubrir la brecha rural-urbana en materia TIC tanto a nivel europeo dentro de las estrategias eEuropa e i2010 como a nivel nacional con el plan Avanza. La recién aprobada Agenda Digital europea contempla de igual manera el logro de la cobertura universal de una banda ancha ultra-rápida en toda la Unión para 2020 (Comisión Europea, 2010b).

A pesar de todo ello, parece claro que en las zonas urbanas y más densamente pobladas suele resultar más sencillo encontrar el apoyo necesario si se tiene algún problema en el manejo de Internet entre amigos, familiares, o simplemente acudiendo a algún ciber-centro público (Liu y San, 2006; Whitacre y Mills, 2007). Esto es lo que explica, según Goolsbee y Klenow (2002), que los individuos residentes en áreas con altos niveles de penetración TIC tengan una mayor probabilidad de ser también usuarios. 
En esta línea, numerosos estudios han confirmado el papel de las externalidades de red en la difusión TIC. Se dice que una tecnología presenta este tipo de externalidades cuando su valor se incrementa con el número de usuarios (Shapiro y Varian, 1999). En el caso de las TIC, es evidente que las posibilidades de comunicación, y con ellas el valor de estas tecnologías aumentan a medida que más y más individuos las utilizan. Uno de los trabajos más recientes que da cuenta de la importancia de tales efectos en la difusión mundial de Internet es el de Andrés et al., (2010) a partir de un panel de 214 países a lo largo de 14 años.

El marco institucional se ha revelado también como otro de los determinantes claves, sobre todo en lo que se refiere al mercado de las telecomunicaciones y, en especial, al mayor o menor grado de competencia en el mismo (Bauer et al., 2002; Estache et al., 2002; Guillén y Suárez, 2005; Andrés et al., 2010). Así, el trabajo de Hargittai (1999) es uno de los primeros que muestra el impacto negativo que tiene la existencia de monopolio en el sector de las telecomunicaciones sobre la difusión de Internet. La evidencia sobre el papel de los precios del sector y la intervención pública en esta materia, sin embargo, todavía no es concluyente (Hargittai, 1999; Andonova, 2006; Chinn y Fairlie, 2007).

Finalmente, en un contexto más amplio y a nivel macro, la calidad de las instituciones con las que cuenta un país (Caselli y Coleman, 2001; Beilock y Dimitrova, 2003; Andonova, 2006; Liu y Sang, 2006; Chinn y Fairlie, 2007, 2010; Billón et al., 2009; Orviska y Hudson, 2009), su nivel de apertura comercial -aunque con evidencia mixta- (Vicente y López, 2006; Chinn y Fairlie, 2010) y algunos elementos culturales, como por ejemplo, las competencias lingüísticas (dominio del inglés) o la presencia de población extranjera (Hargittai, 1999; Beilock y Dimitrova, 2003; Erumban y De Jong, 2006; Schleife, 2010) parecen ser asimimo factores importantes para explicar los patrones de difusión de las TIC.

\section{Marco metodológico y datos}

\subsection{Base de datos}

Los datos para el análisis de la difusión de la Internet móvil en España proceden de la encuesta del INE sobre equipamiento y uso TIC en los hogares para 2010 (INE, 2010b). Esta encuesta viene realizándose desde el año 2002, cuando las autoridades europeas se plantearon la necesidad de contar con estadísticas armonizadas para poder estudiar la penetración de estas tecnologías en los estados miembros (Vicente y López, 2008).

La población objetivo está compuesta por las personas de todas las edades que residen en viviendas familiares principales, si bien sólo los mayores de 15 años son objeto de investigación exhaustiva. La encuesta se realiza de forma personal y telefónica, a partir de un muestreo en tres etapas (secciones censales, viviendas, individuos mayores de 15 años) con estratificación de las unidades de la primera etapa. 
Las viviendas seleccionadas se mantienen en la muestra durante 4 años, configurando un panel rotante en el que se renueva cada año una cuarta parte (INE, 2010a).

La muestra de 2010 está compuesta por 19.384 encuestados. De ese total 16.344 tienen una edad comprendida entre los 16 y 74 años y son éstos los que se consideran relevantes para calcular la tasa de penetración de Internet en España, y por ello, son los que se tendrán en cuenta para las estimaciones.

En la encuesta la pregunta clave para determinar el grado de difusión de la Internet móvil se formula a aquellos individuos que han manifestado previamente haber utilizado Internet en los tres últimos meses. Más concretamente se les pregunta si han empleado alguno de los siguientes dispositivos móviles para el acceso: a) teléfono móvil de banda ancha vía UMTS, 3G, ó 3,5G; b) otro teléfono móvil (GPRS, WAP); c) ordenador de mano (agenda electrónica o similar, PDA, pocket PC, palmtop); d) ordenador portátil fuera de la vivienda habitual o centro de trabajo; o bien e) otros dispositivos. Las respuestas no son excluyentes, y así un individuo podría haber contestado afirmativamente a las cinco opciones consideradas. El Cuadro 1 recoge algunos de los principales resultados de la encuesta en cuanto al uso de estos dispositivos.

\section{CUADRO 1}

\section{ACCESO MÓVIL A INTERNET EN ESPAÑA}

\begin{tabular}{|l|r|}
\hline & \% de individuos \\
\hline Uso de Internet en los tres últimos meses entre la población de 16 a 74 años & 64,2 \\
Acceso móvil a Internet a través de: & 20,3 \\
a) Teléfono móvil de banda ancha vía UMTS, 3G ó 3,5G & 5,5 \\
b) Otro teléfono móvil (GPRS, WAP) & 7,2 \\
c) Ordenador de mano (agenda electrónica o similar, PDA, pocket PC, palmtop) & 24,3 \\
d) Ordenador portátil fuera de la vivienda habitual o centro de trabajo & 1,8 \\
e) Otros dispositivos & 38,4 \\
f) Uso de algún dispositivo móvil & \\
\hline
\end{tabular}

NOTA: Las cifras de acceso móvil se han obtenido teniendo en cuenta el factor de elevación de cada encuestado, y tienen como base la población entre 16 y 74 años que ha manifestado haber utilizado Internet en los tres últimos meses.

FUENTE: Elaboración propia a partir de los microdatos del INE (2010b).

La encuesta del INE revela así que en 2010 un 64 por 100 de la población de 16 a 74 años ha utilizado Internet durante los tres últimos meses. De estos, un 38 por 100 ha accedido a la red a través de algún dispositivo móvil, siendo el ordenador portátil el medio más habitual (24 por 100) seguido por el teléfono móvil de banda ancha (20 por 100), y con una tasa de uso mucho más baja, algún tipo de ordenador de mano (7 por 100). De aquí en adelante se hablará en general de acceso móvil en la medida en que un individiduo haya empleado cualquiera de los dispositivos mencionados para acceder a Internet.

\footnotetext{
2 Pregunta 35 en el cuestionario de la encuesta.
} 


\subsection{Modelo y estimación}

Para analizar la difusión del acceso móvil a Internet en España y, más concretamente, los factores que influyen en la decisión de uso de este tipo de tecnología, el marco metodológico de referencia son los modelos de elección discreta binaria.

En estos modelos la variable dependiente es discreta $\left(Y_{i}\right)$ y toma únicamente dos valores ( 1 si el suceso ocurre y 0 en caso contrario), pudiendo expresarse como una variable continua latente $Y_{i}^{*}$ de la siguiente forma:

$$
Y_{i}^{*}=X_{i}^{\prime} \beta+\mu_{i}
$$

donde sólo $Y_{i}$ es observable, tomando el valor 1 si $Y_{i}^{*} \geq 0$, lo que indicaría, en el caso de análisis, que el individuo i ha utilizado algún dispositivo móvil para acceder a Internet en los tres últimos meses, y 0 en caso contrario. $X_{i}$ sería el vector que recoge las características socio-económicas y demográficas del individuo i, ya que como ha quedado expuesto en el apartado segundo de revisión de la literatura, estos rasgos son claves para explicar la adopción TIC; y $\mu_{i}$ sería el término de error que asumiendo que distribuye normalmente conduce al siguiente modelo probit:

$$
\operatorname{Prob}\left(Y_{i}=1\right)=\phi\left(X_{i}^{\prime} \beta\right)
$$

donde $\phi$ es la función de distribución acumulada de la distribución normal estándar.

Como se ha especificado en el apartado anterior, la información sobre el uso de dispositivos móviles está disponible solamente en relación a los individuos que han manifestado ser usuarios de Internet. Dado que la muestra procede de toda la población, podría introducirse algún tipo de sesgo de selección al estimar el modelo probit para el acceso móvil considerando únicamente los datos de los usuarios de Internet. Para evitar esto, se opta por una estimación en dos etapas (Heckman, 1979; Greene, 1992; Goldfarb y Prince, 2008; Orviska y Hudson, 2009). De esta forma y en primer lugar, se estimará la probabilidad de que un individuo sea usuario de Internet a través de un modelo probit análogo a la expresión [2], y en segundo lugar, para aquéllos que son efectivamente usuarios, se analizará si utilizan dispositivos móviles. Las estimaciones obtenidas en la primera ecuación se utilizarán para construir el inverso del cociente de Mills que introducido como regresor en la segunda ecuación permitirá corregir el posible sesgo de selección. Para estimar esa segunda ecuación será además necesario que alguna de las variables explicativas incluidas en la primera etapa, sea excluida de la segunda (Wooldridge, 2006). En concreto, se trata de excluir aquellas variables que están correlacionadas con la variable dependiente de la primera ecuación pero no con la de la segunda, es decir, correlacionadas con el uso de Internet pero no con el empleo de dispositivos móviles.

El Cuadro 2 recoge la descripción de las variables que van a utilizarse para la estimación de las dos ecuaciones. 


\section{CUADRO 2}

DESCRIPCIÓN DE LAS VARIABLES EMPLEADAS PARA LAS ESTIMACIONES

\begin{tabular}{|c|c|}
\hline Variables dependientes & Descripción \\
\hline Internet & $\begin{array}{l}\text { Dummy con valor } 1 \text { si el individuo (16-74 años) ha utilizado Internet en } \\
\text { los tres últimos meses ( } 0 \text { en otro caso) }\end{array}$ \\
\hline Acceso móvil & $\begin{array}{l}\text { Dummy con valor } 1 \text { si el individuo (16-74 años) ha empleado algún dis- } \\
\text { positivo móvil para acceder a Internet (0 en otro caso) } \\
\text { Base: Usuarios de Internet }\end{array}$ \\
\hline \multicolumn{2}{|l|}{ Variables independientes } \\
\hline Edad & $\begin{array}{l}\text { Edad del encuestado. Variable categórica (1: entre } 16 \text { y } 24 \text { años; } 2 \text { : entre } \\
25 \text { y } 44 \text { años; } 3 \text { : entre } 45 \text { y } 64 \text { años; } 4 \text { : entre } 65 \text { y } 74 \text { años) }\end{array}$ \\
\hline Género & Dummy con valor 1 si el encuestado es mujer (0 en otro caso) \\
\hline Situación laboral & Dummy con valor 1 si el encuestado tiene trabajo ( 0 en otro caso) \\
\hline Nacionalidad & $\begin{array}{l}\text { Nacionalidad del encuestado. Variable categórica (1: española; } 2 \text { : país de } \\
\text { la Unión Europea; 3: otro país) }\end{array}$ \\
\hline Nivel de estudios & $\begin{array}{l}\text { Nivel de estudios terminado. Variable categórica (1: analfabetos o con } \\
\text { estudios primarios; } 2 \text { : estudios secundarios-primera etapa; } 3 \text { : estudios } \\
\text { secundarios-segunda etapa; } 4 \text { : formación profesional de grado superior; } \\
\text { 5: estudios universitarios) }\end{array}$ \\
\hline Ingresos & $\begin{array}{l}\text { Ingresos mensuales netos equivalentes. Variable categórica ( } 1: \text { menos de } \\
600 € ; 2: 600-1.200 € ; 3: 1.200-1.800 € ; 4: \text { más de } 1.800 €)\end{array}$ \\
\hline Hábitat & $\begin{array}{l}\text { Tamaño del municipio. Variable categórica (1: menos de } 10 \text { mil habitan- } \\
\text { tes; } 2: 10-20 \text { mil habitantes; } 3: 20-50 \text { mil habitantes; } 4: 50-100 \text { mil habi- } \\
\text { tantes (no capital de provincia); } 5: \text { más de } 100 \text { mil habitantes (no capita } \\
\text { de provincia); } 6 \text { : capitales de provincia con menos de } 500 \text { mil habitantes; } \\
\text { 7: capitales de provincia con más de } 500 \text { mil habitantes) }\end{array}$ \\
\hline Región & $\begin{array}{l}\text { Región de residencia del encuestado. Variable con } 17 \text { categorías, una por } \\
\text { cada comunidad autónoma. }\end{array}$ \\
\hline
\end{tabular}

NOTA: En el Cuadro 1 se especifican los dispositivos móviles para el acceso a Internet considerados. FUENTE: Elaboración propia.

En la primera ecuación, se considera como variable dependiente una dummy relativa a si el individuo encuestado ha usado Internet en los últimos tres meses. Entre aquéllos que hayan contestado afirmativamente se estudiará el empleo de dispósitivos móviles, tomando como dependiente otra variable dummy con valor 1 en el caso de que el individuo haya usado algún dispositivo móvil para acceder a Internet, ya sea un teléfono móvil de banda ancha u otro tipo de móvil, un ordenador de mano, 
un ordenador portátil fuera de la vivienda habitual o centro de trabajo, o cualquier otro aparato que facilite este acceso móvil ${ }^{3}$.

Como variables independientes, y en consonancia con la revisión de la literatura realizada en el segundo apartado donde se pone de manifiesto la importancia de los factores socio-económicos y demográficos de los individuos para explicar su decisión de uso de este tipo de tecnologías, se incluyen la renta, la edad, el sexo, la situación laboral, la nacionalidad, el nivel de estudios, el tipo de municipio y la región de residencia. También se han considerado las interacciones entre el sexo y la edad con el objetivo de captar si el género tiene efectos diferenciales sobre la probabilidad de usar la Internet móvil según el grupo de edad al que se pertenezca.

De la segunda ecuación se excluyen las variables referidas a la situación laboral y la nacionalidad ya que, como se ha comentado anteriomente, la estimación en dos etapas corrigiendo por un posible sesgo de selección muestral exige no incluir en la segunda ecuación al menos alguna de las variables explicativas consideradas en la primera. En concreto, han de excluirse aquellas variables que estando correlacionadas con el uso de Internet no lo estén con el empleo de dispositivos móviles. La exclusión de la nacionalidad responde a la falta de observaciones suficientes para considerarla en la segunda ecuación, mientras que no se tiene en cuenta la situación laboral en consonancia con lo que han hecho otros autores que previamente han abordado el tema. Goldfarb y Prince (2008), al estudiar la conexión a Internet en el hogar y el uso que se hace desde ella, excluyen de la segunda de las ecuaciones toda una serie de variables relacionadas con la actividad laboral del individuo (se trae trabajo a casa, lleva un negocio desde casa, etc.) así como la variable referente a la presencia de hijos en el hogar. Orviska y Hudson (2009), con un modelo similar al de Goldfarb y Prince (2008), para explicar el uso de Internet y los servicios que se emplean online, no tienen en cuenta la situación laboral, el estado civil y el nivel de estudios del individuo. A diferencia de estos autores, en el presente análisis de la Internet móvil sí que se incluye el nivel educativo puesto que la evidencia previa incide en su impacto fundamental en el uso de este tipo de tecnología (Akiyoshi y Ono, 2008; Smith, 2010).

Inicialmente se había contemplado la posibilidad de incluir en la segunda ecuación, la relativa al acceso móvil, una variable indicativa de las competencias digitales de los encuestados, tal como hacen Akiyoshi y Ono (2008), que consideran la rapidez que los individuos tienen para procesar textos en el ordenador como indicador de tales competencias. En la encuesta del INE, con el objetivo de medir los conocimientos informáticos de los individuos, se les pregunta acerca de las tareas

\footnotetext{
3 Aunque podría haberse optado por estudiar el acceso móvil según el tipo de dispositivo empleado, se ha preferido considerar de forma agregada este tipo de acceso dados los bajos niveles de uso que todavía existen en España y que conducirían a modelos donde el éxito es poco frecuente. Así, sólo el 24,3 por 100 de los usuarios de Internet emplean un ordenador portátil para acceder de forma móvil a Internet, lo que representa sobre el total de la población de 16 a 74 años una tasa de tan sólo el 15 por 100 .
} 
relacionadas con Internet que han realizado (usar un buscador, usar aplicaciones para compartir ficheros, etcétera). Sin embargo, esta variable no ha sido incluida en la estimación final por la sospecha de doble causalidad: así, cuantas más habilidades digitales tiene un individuo más probable es que utilice dispositivos móviles porque le resulta más sencillo, pero al mismo tiempo el uso de este tipo de acceso puede favorecer que se realicen más de estas tareas.

Por otro lado, es preciso señalar las dos siguientes cuestiones respecto a la utilización de la variable ingresos. En primer lugar, esta variable recoge los ingresos mensuales equivalentes, es decir, aquéllos resultantes de aplicar una escala de equivalencia a la renta con el objetivo de ajustarla en función del tamaño y composición del hogar. La información disponible en la encuesta del INE sobre esta variable hace referencia al total mensual neto del hogar encuestado. Dado que se conoce el tamaño de los hogares y su composición por edades, y que los modelos se plantean en términos individuales, es decir, que un individuo sea o no usuario (móvil) de Internet, los ingresos se han ajustado utilizando la escala de equivalencia modificada de la OCDE, que asigna valor 1 al primer adulto, 0,5 a los demás integrantes del hogar mayores de 14 años y 0,3 a los menores de esa edad (OCDE, 2011). En segundo lugar, la variable ingresos presenta un porcentaje bastante elevado de casos perdidos en la encuesta (22 por 100). Para evitar la introducción de sesgos por la ausencia de respuesta en un factor tan crucial como éste para explicar la difusión TIC, se ha procedido a aplicar el método de imputación múltiple (Little y Rubin, 2002).

Cabe además señalar que, en la encuesta del INE, en la codificación del nivel de estudios las categorías analfabetos y estudios primarios aparecen separadas. Para la estimación de los modelos, estas dos categorías se han agrupado ya que el número de casos dentro de la primera es muy bajo lo que planteaba algunos problemas. En concreto, en la muestra solamente dos individuos analfabetos indicaban que habían utilizado Internet en los tres últimos meses y de esos dos, ninguno había empleado dispositivos móviles.

Por último, mencionar que en las variables cualitativas se considerará como categoría de referencia siempre la primera, esto es, en el nivel de estudios aquellos individuos analfabetos o con estudios primarios, los que tienen unos ingresos mensuales inferiores a los 600 euros, los menores de 25 años, que residen en municipios con menos de 10 mil habitantes, hombres, españoles, y parados e inactivos. La categoría de referencia para la variable región será Andalucía (la primera en orden alfabético).

\section{Resultados}

El Cuadro 3 recoge los resultados de las estimaciones correspondientes a los dos modelos probit planteados para explicar, en primer lugar, el hecho de que un individuo sea usuario de Internet y, en segundo lugar, entre éstos, el que se utilice algún dispositivo móvil para el acceso. 
En términos generales, se observa que la gran mayoría de los factores explicativos considerados son significativos, y presentan el efecto esperado.

En la primera ecuación los coeficientes referentes a las variables estudios, ingresos, situación laboral y tamaño del municipio son todos ellos positivos y significativos; mientras que la edad y la procedencia de un país extracomunitario tienen una asociación negativa y significativa con el uso de Internet. Por tanto, la probabilidad de utilizar esta tecnología aumentaría con el nivel de renta y estudios, el tamaño del municipio en el que se reside y para los ocupados respecto a los parados e inactivos; por el contrario, esta probabilidad disminuiría a medida que aumenta la edad y para los extranjeros que proceden de fuera de la Unión Europea respecto a los individuos con nacionalidad española. Los signos negativos de las variables relativas al género $\mathrm{y}$ a sus interacciones con la edad indican una menor probabilidad de uso de Internet de las mujeres frente a los hombres (incluso dentro del mismo grupo de edad), sin embargo tales efectos no son significativos ${ }^{4}$.

Todos estos resultados, aunque en la línea de la evidencia previa que hay para otros países y que se ha revisado en el apartado segundo, tienen como implicación fundamental que aún existe una brecha socio-económica y demográfica muy importante en el uso de Internet en España, a pesar de que los niveles de penetración superan ya el 60 por 100 de la población. En concreto, son la renta y el nivel educativo los factores que marcan las mayores diferencias, y especialmente, el hecho de que una persona haya alcanzado el nivel de educación universitaria. Esto confirma el papel que el capital humano viene desempeñando en la difusión de las TIC y cuya importancia se acentúa a medida que más y más contenido está disponible en Internet, y los servicios y aplicaciones se van sofisticando.

Asimismo cabe mencionar que, a diferencia del estudio de ONTSI (2009) $)^{5}$ sobre el perfil demográfico de los internautas y en el que se concluye, en base a un análisis descriptivo, que el tamaño del municipio donde un invididuo reside no es un elemento fundamental a la hora de explicar el uso de Internet y que sólo existe una marcada brecha entre los extremos, esto es, entre las grandes capitales con más de 500 mil habitantes y los pequeños municipios con menos de 10 mil, los resultados del presente análisis muestran una asociación positiva y significativa entre estas dos variables: los individuos que residen en municipios más poblados tienen una mayor probabilidad de utilizar Internet en comparación con los que viven en zonas rurales. Este hecho puede venir explicado por dos razones fundamentales: por un lado, porque en las zonas urbanas las conexiones sean mejores y haya más opciones de conexión a Internet desde centros públicos cercanos al domicilio (bibliotecas, ciber-centros, etcétera); y por otro lado, la relevancia del tamaño del hábitat puede ser indicativa de la presencia de externalidades de red y de ciertos efectos de aprendi-

${ }^{4}$ La variable relativa al género resulta significativa cuando se introduce sin considerar sus interacciones con la edad. Una vez tales efectos se incorporan a la ecuación, su importancia desaparece.

5 ONTSI es el Observatorio Nacional de las Telecomunicaciones y de la Sociedad de la Información http://www.ontsi.red.es/index.action. 
zaje social. Como se ha comentado en el segundo apartado, varios trabajos en este campo han puesto de manifiesto que en los entornos urbanos resulta más sencillo encontrar el apoyo necesario cuando se presentan problemas en el manejo de estas tecnologías (Whitacre y Mills, 2007; Schleife, 2010).

En relación con estos efectos, se pueden señalar además los coeficientes positivos y significativos de Aragón, Baleares, Castilla-La Mancha, Cataluña, Comunidad Valenciana y Madrid; mientras que el coeficiente asociado a Galicia es también significativo pero negativo ${ }^{6}$.

Por otro lado, se detecta que dentro del colectivo de los inmigrantes no todos tienen el mismo nivel de uso de Intenet, ya que el impacto de la variable nacionalidad difiere según el país de origen. Así, cuando el individuo procede de un país de la Unión Europea el efecto es positivo, mientras que resulta negativo en el caso de los extracomunitarios. No obstante, sólo este último resultado es significativo. Sinai y Waldfogel (2004), argumentan la mayor tendencia de uso de Internet entre la población extranjera tanto por motivos de mantener los contactos con los familiares y amigos del país de origen como para superar problemas de aislamiento social. Nuestros resultados indican que esto depende del país de procedencia del inmigrante. $\mathrm{La}$ menor probabilidad de uso de los individuos extracomunitarios refleja posiblemente toda una combinación de factores estructurales relacionados con menores niveles educativos y de renta de sus países de origen, y quizás también alguna dificultad lingüística como apunta Schleife (2010), aunque en la encuesta del INE no se dispone de información suficiente como para poder valorar tales cuestiones, ya que no se indica el país concreto de procedencia del inmigrante.

Aunque podría sospecharse de la existencia de problemas derivados de asociaciones fuertes entre algunas de las variables explicativas como, por ejemplo, el hecho de ser extranjero y el nivel de estudios o la renta, el grado de relación entre estos factores es bajo. Así, la V de Cramer toma unos valores bastante próximos a cero en los dos casos comentados, en concreto, 0,13 y 0,05 , respectivamente. Además la exclusión de la nacionalidad de la estimación no afecta ni a los niveles de significación ni a los coeficientes de las restantes variables.

En lo que se refiere al acceso móvil, el aspecto fundamental sobre el que cabe llamar la atención es el hecho de que el perfil socio-económico y demográfico de los individuos sigue resultando relevante para explicar el uso de estos dispositivos a pesar de que se controla por tales factores en la primera ecuación. Así, la edad mantiene su impacto negativo; mientras que el nivel educativo y los ingresos siguen presentando asociaciones positivas y significativas; el género ahora sí ejerce una influencia significativa y sólo dejaría de ser relevante el tamaño del hábitat.

Por tanto, los resultados muestran que, entre los usuarios de Internet, los que usan dispositivos móviles para el acceso son aquéllos de menor edad, los hombres frente a las mujeres, con mayor nivel educativo y mayores ingresos.

\footnotetext{
${ }^{6}$ Recordemos que se ha tomado Andalucía como categoría de referencia.
} 
Como primer elemento a destacar habría que llamar la atención sobre el coeficiente significativo y positivo que se obtiene para la renta y que contrasta con los resultados de Akiyoshi y Ono (2008), quienes no encuentran evidencia significativa para este factor. Para entender el impacto clave que tiene la renta en el caso español es preciso tener en cuenta varios elementos. Por un lado, el acceso móvil considerado en el presente análisis incluye cualquier dispositivo que permita este tipo de acceso (ordenadores portátiles, teléfonos móviles, agendas electrónicas) mientras que Akiyoshi y Ono (2008) se centran en el caso del acceso a Internet a través de los teléfonos móviles en Japón. De aquí que el resultado obtenido puede estar reflejando en cierta medida el mayor coste que, en general, supone la adquisición de un ordenador frente a un teléfono. Además, es preciso considerar la diferencia temporal entre los datos de los dos trabajos, 2001 frente a 2010, un periodo de casi una década donde se han sucedido generaciones de telefonía móvil y se han popularizado nuevos dispositivos de acceso móvil. Así, por ejemplo, 2010 ha sido el año del ipad y del iphone 4. Los datos del informe Pew (Smith, 2010) para los Estados Unidos sí que muestran cómo las tasas de uso de la Internet móvil se incrementan a medida que aumenta la renta si bien también se detectan diferencias en los patrones de uso por tipo de dispositivo. En concreto, se observa que la conexión a Internet a través del teléfono móvil es mucho más frecuente entre colectivos tradicionalmente desfavorecidos como los latinos y afro-americanos frente a la población de origen europeo. Por otro lado, habría que considerar los costes de la conexión móvil a Internet. Según los datos de la OCDE (2009a), para un tráfico mensual entre los 20 y 1.000 megabytes que permitiría básicamente consultar el correo electrónico y navegar por las diversas páginas de Internet, España tiene el precio mensual medio para la banda ancha móvil más alto entre los países miembros: 33,29 dólares americanos en paridad de poder adquisitivo frente a los 10,98 de Suecia ${ }^{7}$. Además, el trabajo de West (2009) para la institución Brookings sobre las comunicaciones móviles en cuatro países (España, Estados Unidos, Gran Bretaña, y Japón) indica que los encuestados españoles son los que manifiestan en mayor grado que la principal razón para no descargarse aplicaciones en el teléfono móvil son los costes.

Este mismo trabajo apunta otro hecho interesante que puede relacionarse con el efecto positivo y significativo del nivel de estudios sobre el acceso móvil. Así, según los resultados de West (2009), los españoles también son, entre la poblacion de los cuatros países considerados, los que ven en mayor medida los teléfonos móviles como una extensión de sus ordenadores. En este sentido, trabajar con un ordenador requiere siempre una serie de habilidades que suelen están presentes en las personas con mayores niveles educativos. De hecho, Akiyoshi y Ono (2008) encuentran evidencia significativa de que tanto las competencias digitales como el nivel educativo son elementos claves para el acceso móvil a Internet.

\footnotetext{
${ }^{7}$ Desde marzo de 2010 las tarifas máximas a aplicar en caso de conexiones móviles a Internet desde el extranjero están limitadas por la Unión Europea.
} 
Las asociaciones negativas y significativas que se observan tanto para la edad como para el hecho de ser mujer con el uso móvil podrían explicarse por una combinación de factores. Por un lado, pueden estar reflejando cierta menor preferencia de las personas de mayor edad y de las mujeres por los gadgets tecnológicos (Dholakia, 2006). Por otro lado, para el caso de los mayores, el impacto significativo puede estar indicando problemas de accesibilidad en el diseño de este tipo de dispositivos. Tal como señalan Vicente y López (2010), el diseño de algunos de los últimos modelos de teléfonos móviles y mini-ordenadores con teclados comprimidos o pantallas táctiles plantean dificultades de uso para las personas de mayor edad así como para aquéllos con alguna discapacidad. Los resultados de las interacciones entre la edad y el sexo muestran además que la brecha de género es significativa aún cuando se controla por la edad. Así, las mujeres más jóvenes tienen una menor probabilidad de uso de la Internet móvil que los varones jóvenes.

En cuanto a los efectos geográficos, mientras que el tamaño del hábitat deja de ser significativo, se detectan asociaciones significativas y positivas para 10 de las 17 regiones españolas, con la excepción de Canarias que presenta un coeficiente también significativo pero negativo.

Finalmente, no parece haber sesgo de selección muestral ya que el coeficiente estimado para el inverso del cociente de Mills no es significativo, por lo que las dos ecuaciones podrían haberse estimado de forma independiente obteniendo resultados análogos. 


\section{CUADRO 3}

ESTIMACIONES PROBIT PARA EL USO DE INTERNET Y DE DISPOSITIVOS MÓVILES EN EL ACCESO

\begin{tabular}{|c|c|c|}
\hline & Uso de Internet & Acceso móvil \\
\hline Edad (25-44 años) & $-1,204(-15,34) * * *$ & $-0,398(-7,13)^{* * *}$ \\
\hline Edad (45-64 años) & $-1,920(-24,69) * * *$ & $-0,847(-13,63) * * *$ \\
\hline Edad (65-74 años) & $-2,609(-28,35)^{* * *}$ & $-1,297(-9,72) * * *$ \\
\hline Género (mujer) & $-0,041(-0,38)$ & $-0,131(-1,92)^{*}$ \\
\hline Situación laboral (ocupados) & $0,360(12,09)^{* * *}$ & \\
\hline Nacionalidad_extranjeroUE & $0,002(0,02)$ & \\
\hline Nacionalidad_otros & $-0,330(-5,72)^{* * *}$ & \\
\hline Estudios_Secundarios & $0,563(15,94)^{* * *}$ & $0,008(0,12)$ \\
\hline Estudios_Secundarios & $1,183(30,58)^{* * *}$ & $0,326(5,15)^{* * *}$ \\
\hline Formación Profesional & $1,472(28,3)^{* * *}$ & $0,369(5,33)^{* * *}$ \\
\hline Estudios Universitarios & $2,001(39,38) * * *$ & $0,506(7,70)^{* * *}$ \\
\hline Ingresos $(600-1.200 € /$ mes $)$ & $0,260(7,62)^{* * *}$ & $0,055(1,34)$ \\
\hline Ingresos $(1.200-1.800 € /$ mes $)$ & $0,442(9,71)^{* * *}$ & $0,275(5,93)^{* * *}$ \\
\hline Ingresos (más de $1.800 € /$ mes) & $0,642(7,71) * * *$ & $0,441(7,02)^{* * *}$ \\
\hline Municipio (10-20 mil hab.) & $0,119(2,79) * * *$ & $-0,020(-0,41)$ \\
\hline Municipio (20-50 mil hab.) & $0,186(4,1) * * *$ & $0,005(0,10)$ \\
\hline Municipio (50-100 mil hab.) & $0,094(1,73)^{*}$ & $0,068(1,13)$ \\
\hline Municipio (no c.p., más de 100 mil hab.) & $0,228(3,92)^{* * *}$ & $-0,056(-0,88)$ \\
\hline Municipio (c.p., menos de 500 mil hab.) & $0,242(6,1)^{* * *}$ & $0,050(1,17)$ \\
\hline Municipio (c.p. más de 500 mil hab.) & $0,260(4,69)^{* * *}$ & $0,063(1,12)$ \\
\hline Mujer* Edad (25-44 años) & $-0,024(-0,21)$ & $-0,203(-2,59) * * *$ \\
\hline Mujer* Edad (45-64 años) & $-0,046(-0,4)$ & $-0,205(-2,36) * *$ \\
\hline Mujer* Edad (65-74 años) & $-0,140(-1,04)$ & $0,262(1,33)$ \\
\hline Aragón & $0,238(3,29)^{* * *}$ & $0,156(2,06)^{* *}$ \\
\hline Asturias & $0,077(1,35)$ & $0,178(2,77)^{* * *}$ \\
\hline Baleares & $0,479(5,78) * * *$ & $0,040(0,46)$ \\
\hline Canarias & $0,037(0,54)$ & $-0,321(-3,80) * * *$ \\
\hline Cantabria & $0,063(0,7)$ & $0,129(1,30)$ \\
\hline Castilla-León & $0,053(0,81)$ & $0,303(4,15)^{* * *}$ \\
\hline Castilla-La Mancha & $0,254(3,48)^{* * *}$ & $0,237(3,08)^{* * *}$ \\
\hline Cataluña & $0,556(10,09) * * *$ & $0,088(1,53)$ \\
\hline Ceuta y Melilla & $-0,135(-0,95)$ & $0,280(1,93) *$ \\
\hline Comunidad Valenciana & $0,179(3,11)^{* * *}$ & $0,249(3,99) * * *$ \\
\hline Extremadura & $0,111(1,47)$ & $0,317(3,61)^{* * *}$ \\
\hline Galicia & $-0,130(-2,02)^{* *}$ & $0,446(6,07)^{* * *}$ \\
\hline Madrid & $0,160(2,45)^{* *}$ & $0,276(4,19)^{* * *}$ \\
\hline Murcia & $-0,069(-0,91)$ & $0,338(4,01)^{* * *}$ \\
\hline Navarra & $0,052(0,97)$ & $-0,025(-0,42)$ \\
\hline País Vasco & $0,111(1,63)$ & $-0,097(-1,33)$ \\
\hline Rioja & $0,090(0,97)$ & $0,115(1,15)$ \\
\hline Inverso del cociente de Mills & & $-0,007(-1,06)$ \\
\hline Constante & $0,364(4,56)^{* * *}$ & $-0,339(-4,12) * * *$ \\
\hline & 16.344 & 9.152 \\
\hline F-test & $142,14 * * *$ & $23,59 * * *$ \\
\hline
\end{tabular}

NOTAS: La tabla recoge las estimaciones de los coeficientes de las variables y, entre paréntesis, el valor del estadístico t. Los asteriscos $* * *, * *, *$ indican significativo a los niveles del 1 por 100,5 por 100 , y 10 por 100 , respectivamente. Se ha usado «c.p» como abreviatura de capital de provincia. Categorías de referencia: menores de 25 años; hombres; parados e inactivos; nacionalidad española; analfabetos o con estudios primarios; ingresos menores de $600 € / \mathrm{mes}$; municipio de menos de 10 mil habitantes; y Andalucía FUENTE: Elaboración propia. 


\section{Conclusiones}

El objetivo de este trabajo ha consitido en realizar una primera aproximación al fenómeno de la Internet móvil en España. Si bien en otros países han comenzado a realizarse estudios sobre el uso de dispositivos digitales móviles (teleléfonos, ordenadores portátiles, agendas electrónicas, etc.) para acceder a Internet, en España este es un campo que aún no se había abordado.

$\mathrm{El}$ análisis se ha planteado en dos etapas, primero estimando la probabilidad que tiene un individuo de ser usuario de Internet, y segundo, para los que efectivamente utilizan Internet, se ha estudiado si emplean dispositivos móviles.

Los resultados muestran que a pesar de que el porcentaje de población que utiliza Internet de forma regular (en los tres últimos meses) supera ya el 60 por 100, aún persiste una brecha socio-económica y demográfica muy importante en el uso de esta tecnología en España. Así, existen claras y significativas asociaciones negativas con la edad, y la nacionalidad. La asociación también es significativa pero positiva para los casos de la renta, el nivel de estudios, la situación laboral (tener empleo) y el tamaño del municipio en el que se reside.

En lo que se refiere al acceso móvil a Internet, vuelve a repetirse este patrón socio-económico y demográfico. Así, entre los que ya son usuarios de Internet, los que emplean dispositivos móviles para el acceso son aquéllos de menor edad, los hombres frente a las mujeres, con mayor nivel educativo, y mayores ingresos.

Toda esta evidencia apunta a la necesidad de reforzar las acciones destinadas a fomentar el uso de Internet entre la población, con acciones especiales dirigidas a los colectivos de mayor riesgo de exclusión digital. Aunque durante los últimos años se han ido poniendo en marcha distintas medidas en esta dirección, el análisis realizado en este trabajo con los datos de la última encuesta del INE muestra que las desigualdades digitales persisten en el simple uso de Internet y, más aún, se extienden hacia las nuevas formas de acceso que muy posiblemente definirán la Internet del futuro.

\section{Referencias bibliográficas}

[1] AKIYOSHI, M. y ONO, H. (2008): «The diffusion of mobile internet in Japan», The Information Society, n. ${ }^{\circ}$ 24, pp. 292-303.

[2] ANDONOVA, V. (2006): «Mobile phones, the internet and the institutional environment», Telecomunications Policy, n. ${ }^{\circ} 30$, pp. 29-45.

[3] ANDRÉS, L.; CUBERES, D.; MAME, D. y SEREBRISKY, T. (2010): «The diffusion of the internet: a cross-country analysis», Telecommunications Policy, n. ${ }^{\circ} 34$, pp. 323340 .

[4] BAUER, J. M.; BERNE, M. y MAITLAND, C.F. (2002): «Internet access in the European Union and in the United States», Telematics and Informatics, n. ${ }^{\circ}$ 19, pp. 117-137.

[5] BEILOCK, R. y DIMITROVA, D. V. (2003): «An exploratory model of inter-country internet diffusion», Telecommunications Policy, n. . 27, pp. 237-252. 
[6] BILLÓN, M.; EZCURRA, R. y LERA-LÓPEZ, F. (2008): «The spatial distribution of the internet in the European Union. Does geographical proximity matter?», European Planning Studies n. ${ }^{\circ}$ 16, pp.119-142.

[7] BILLÓN, M., MARCO, R. y LERA-LÓPEZ, F. (2009): «Disparities in ICT adoption. A multidimensional approach to study the cross-country digital divide», Telecommunications Policy, n. ${ }^{\circ}$ 33, pp. 596-610.

[8] CASELLI, F. y COLEMAN, W. J. (2001): «Cross-country technology diffusion: the case of computers», American Economic Review, n. ${ }^{\circ}$ 91, pp. 328-335.

[9] CHINN, M.D. y FAIRLIE, R.W. (2007): «The determinants of the global divide: a cross-country analysis of computer and internet penetration», Oxford Economic Papers, n. ${ }^{\circ} 59$, pp.16-44.

[10] CHINN, M. D. y FAIRLIE, R. W. (2010): «ICT use in the developing world: an analysis of differences in computer and internet penetration», Review of International Economics, n. ${ }^{\circ} 18$, pp.153-167.

[11] COMISIÓN EUROPEA (2008): Future networks and the internet (COM/2008/594 final), Comisión Europea, Bruselas.

[12] COMISIÓN EUROPEA (2010a): Broadband access in the EU: situation at 1 July 2010, Communications Committee Working Paper, DG INFSO/C4 COCOM10-29, Comisión Europea, Bruselas.

[13] COMISIÓN EUROPEA (2010b): Una agenda digital para Europa (COM/2010/ 0245f2), Comisión Europea, Bruselas.

[14] DASGUPTA, S.; LALL, S. y WHEELER, D. (2005): «Policy reform, economic growth and the digital divide: an econometric analysis», Oxford Development Studies, n. ${ }^{\circ} 33$, pp. 229-243.

[15] DEMOUSSIS, M. y GIANNAKOPOULOS, N. (2006): «Facets of the digital divide in Europe: determination and extent of internet use», Economics of Innovation and New Technology, n. ${ }^{\circ}$ 15, pp. 235-246.

[16] DHOLAKIA, R. R. (2006): «Gender and IT in the household: evolving patterns of internet use in the United States», The Information Society Journal, n. ${ }^{\circ}$ 22, pp. 231-240.

[17] DIMAGGIO, P.; HARGITTAI, E.; CELESTE, C. y SHAFER, S. (2004): «Digital inequality: from unequal access to differentiated use», en K. Neckerman (ed.): Social Inequality, Russell Sage Foundation, New York, pp.355-400.

[18] ERUMBAN, A. A. y DE JONG, S. B. (2006): «Cross-country differences in ICT adoption: a consequence of culture?», Journal of World Business, n. ${ }^{\circ} 41$, pp. 302-314.

[19] ESTACHE, A.; MANACORDA, M. y VALLETTI, T. M. (2002): «Telecommunication reforms, access regulation and internet adoption in Latin America», Economía, n. ${ }^{\circ} 2$, pp. 153-217.

[20] FAIRLIE, R. W. (2004): «Race and the digital divide», Contributions to Economic Analysis \& Policy, n. ${ }^{\circ}$ 3, pp.1-15.

[21] GOLDFARB, A. y PRINCE, J. (2008): «Internet adoption and usage patterns are different: implications for the digital divide», Information Economics and Policy, n. ${ }^{\circ} 20$, pp. $2-15$.

[22] GOOLSBEE, A. y KLENOW, P. J. (2002): «Evidence on learning and network externalities in the diffusion of home computers», The Journal of Law and Economics, $\mathrm{n}$. 45, pp. 317-344. 
[23] GREENE, W. H. (1992): «A statistical model for credit scoring», Department of Economics Working Paper, 92-29, Leonard N. Stern School of Business, New York.

[24] GRUBESIC, T.H. (2006): «A spatial taxonomy of broadband regions in the United States», Information Economics and Policy, n. ${ }^{\circ} 18$, pp. 423-448.

[25] GUILLÉN, M. F. y SUÁREZ, S. (2005): «Explaining the global digital divide: economic, political and sociological drivers of cross-national internet use», Social Forces, n. ${ }^{\circ}$ 84, pp. 681-708.

[26] HARGITTAI, E. (1999): «Weaving the western web: explaining differences in internet connectivity among OECD countries», Telecommunications Policy, n. ${ }^{\circ}$ 23, pp. 701-718.

[27] HECKMAN, J.J. (1979): «Sample selection bias as a specification error», Econometrica, n. ${ }^{\circ} 47$, pp. $153-161$.

[28] HELSPER, E. J. y EYNON, R. (2010): «Digital natives: where is the evidence?», British Educational Research Journal, n. . 36, pp. 503-520.

[29] HILBERT, M. (2010): «When is cheap, cheap enough to bridge the digital divide? Modeling income related structural challenges of technology diffusion in Latin America», World Development, n. ${ }^{\circ} 38$, pp.756-770.

[30] HORRIGAN, J. B.; STOLP, C. y WILSON, R.H. (2006): «Broadband utilization in space: effects of population and economic structure», The Information Society, n. ${ }^{\circ} 22$, pp. 341-356.

[31] INE (2010a): Encuesta sobre equipamiento y uso TIC en los hogares 2010. Informe metodológico. Disponible en: http://www.ine.es/

[32] INE (2010b): Encuesta sobre equipamiento y uso TIC en los hogares 2010. Microdatos. Disponible en: http://www.ine.es/

[33] ISHII, K. (2004): «Internet use via mobile phone in Japan», Telecommunications Policy, n. ${ }^{\circ}$ 28, pp.43-58.

[34] ITU (2006): World telecommunication/ICT development report 2006. Measuring ICT for social and economic development, ITU, Geneva.

[35] KARSHENAS, M. y STONEMAN, P. (1995): «Technological diffusion», en P. Stoneman (Ed.): Handbook of the Economics of Innovation and Technological Chang, Blackwell, Oxford, pp. 263-297.

[36] KIISKI, S. y POHJOLA, M. (2002): «Cross-country diffusion of the internet», Information Economics and Policy, n. ${ }^{\circ} 14$, pp. 297-310.

[37] LITTLE, R. J. A. y RUBIN, D. B. (2002): Statistical analysis with missing data, Wiley Interscience, J. Wiley \& Sons, Hoboken, New Jersey.

[38] LIU, M.-C. y SAN, G. (2006): «Social learning and digital divides: a case study of internet technology diffusion», Kyklos, n. ${ }^{\circ}$ 59, pp. 307-321.

[39] NORRIS, P. (2001): Digital divide: Civic engagement, information poverty, and the internet worldwide, Cambridge University Press, New York.

[40] OCDE (2009a): Communications Outlook 2009, OCDE, Paris.

[41] OECD (2009b): Guide to measuring the information society, 2009, OCDE, Paris.

[42] OCDE (2011): What are Equivalence Scales? Disponible en: http://www.oecd.org/ Long/Abstract/0,3425,en_2649_33933_35411112_1_1_1_1,00.html.

[43] ONTSI (2009): Perfil sociodemográfico de los internautas- Análisis de datos INE 2009. Disponible en: http://www.ontsi.red.es/hogares-ciudadanos/articles/id/4343/perfilsociodemografico-los-internautas-analisis-datos-ine-2009.html 
[44] ORVISKA, M. y HUDSON, J. (2009): «Dividing or uniting Europe? Internet usage in the EU», Information Economics and Policy n. ${ }^{\circ} 21$, pp. 279-290.

[45] POHJOLA, M. (2003): «The adoption and diffusion of ICT across countries: patterns and determinants», en D.C. Jones (Ed.): The New Economy Handbook, Academia Press, San Diego, pp. 77-100.

[46] PRENSKY, M. (2001): «Digital natives, digital immigrants. Part 1», On the Horizon, n. ${ }^{\circ}$, pp. 1-6.

[47] ROGERS, E. M. (2003): Diffusion of innovations (5th ed.), The Fress Press, New York.

[48] SCHLEIFE, K. (2010): «What really matters: regional versus individual determinants of the digital divide in Germany», Research Policy, n. ${ }^{\circ}$ 39, pp.173-185.

[49] SHAPIRO, C. y VARIAN, H. R. (1999): Information rules, Harvard Business School Press, Cambridge.

[50] SINAI, T. y WALDFOGEL, J. (2004): «Geography and the internet: is the internet a substitute or a complement for cities?», Journal of Urban Economics, n. ${ }^{\circ}$ 56, pp. 1-24.

[51] SMITH, A. (2010): Mobile access 2010, Pew Internet \& American Life Project, Washington. Disponible en: http://pewinternet.org/Reports/2010/Mobile-Access-2010.aspx

[52] US DEPARTMENT OF COMMERCE (2000): Falling through the Net: toward digital inclusion, US Department of Commerce, Washington DC.

[53] US DEPARTMENT OF COMMERCE (2010): Exploring the digital nation: home broadband internet adoption in the United States, US Department of Commerce, Washington DC.

[54] VICENTE, M. R. y LÓPEZ, A.J. (2006): «Patterns of ICT diffusion across the European Union», Economic Letters, n. ${ }^{\circ}$ 93, pp. 45-51.

[55] VICENTE, M. R. y LÓPEZ, A. J. (2008): «Métricas e indicadores de la sociedad de la información: panorámica de la situación actual», Estadística Española, n. ${ }^{\circ}$ 50, pp. 273 320.

[56] VICENTE, M. R. y LÓPEZ, A. J. (2010): «A multidimensional analysis of the disability digital divide: some evidence for internet use», The Information Society Journal, $\mathrm{n} .{ }^{\circ}$ 26, pp. 48-64.

[57] WEST, D. M. (2009): What consumers want from mobile communications in the United States, United Kingdom, Spain, and Japan, Governance Studies at Brookings. Disponible en: http://www.brookings.edu/ /media/Files/rc/papers/2009/09_mobile_west/09_ mobile_west.pdf

[58] WHITACRE, B. E. y MILLS, B. F. (2007): «Infrastructure and the rural-urban divide in high-speed residential internet access», International Regional Science Review, n. ${ }^{\circ}$ 30, pp.249-273.

[59] WOOLDRIDGE, J. M. (2006): Introductory econometrics. A modern approach, SouthWestern, Mason, Ohio. 